\title{
LncRNA TP73-AS1 interacted with miR-141-3p to promote the proliferation of non-small cell lung cancer
}

\author{
Xinfa Liu ${ }^{1}$, Mingming Wang ${ }^{2}$, Yanzhi Cui ${ }^{3}$
}

1Department of Respiration, Hebei Provincial Hospital of Traditional Chinese
Medicine, Shi Jiazhuang, China
'Department of Clinical Laboratory, Qingdao Woman and Children's Hospital,
Qingdao, China
${ }^{3}$ Tumour Institute, Fourth Hospital of Hebei Medical University, Shi Jiazhuang, China

Submitted: 6 May 2017

Accepted: 25 July 2017

Arch Med Sci 2019; 15 (6): 1547-1554

DOI: https://doi.org/10.5114/aoms.2019.86820

Copyright @ 2019 Termedia \& Banach

\section{Abstract}

Introduction: Recent studies have shown that long non-coding RNAs (IncRNAs) are involved in a variety of biological processes and diseases in humans, including cancer. However, the exact effects and molecular mechanisms of TP73-AS1 in non-small cell lung cancer (NSCLC) progression are still unknown. The present study is aimed to reveal the detailed functions and the mechanism of TP73-AS1 in the regulation of NSCLC cell proliferation.

Material and methods: TP73-AS1 expression in NSCLC tissues and cell lines was determined using real-time PCR assays. The functions of TP73-AS1 in the regulation of NSCLC cell proliferation was evaluated using BrdU assays. The interaction between TP73-AS1 and miR-141-3p was confirmed using luciferase report gene assays.

Results: TP73-AS1 was upregulated in NSCLC tissues and cell lines. However, when knockdown of TP73-AS1 inhibited the NSCLC proliferation. By using online tools, we screened out miR-141-3p may combined with TP73-AS1. With use of luciferase assays, we confirmed that miR-141-3p could directly bind to TP73-AS1. In NSCLC tissues, miR-141-3p was down-regulated; TP73-AS1 was inversely correlated with miR-141-3p.

Conclusions: Our data suggest that TP73-AS1 might be an oncogenic IncRNA that promotes proliferation of NSCLC and might be regarded as a therapeutic target in NSCLC.

Key words: long non-coding RNAs, non-small cell lung cancer, miR-141-3p, proliferation.

\section{Introduction}

Lung cancer is the leading cause of cancer-related death worldwide. Non-small cell lung cancer (NSCLC) constitutes approximately $80 \%$ of all diagnosed lung cancers cases, and the 5 -year survival rate of the disease is $\sim 20 \%$ [1]. Although surgical operation still represents the main curative treatment for NSCLC up to stage IIIA disease, the risk of postoperative disease recurrence has been reported to be as high as 52\% [2]. Most patients eventually succumb to recurrence of the disease despite the high initial response to therapy. According to the results of several large-sample clinical studies on the effect of surgical operation of global NSCLC patients, the five-year survival rate is about 31-42\% [3]. Recently, advances in clinical and experimental oncology have been made for treating NSCLC, but its

\author{
Corresponding author: \\ Dr. Yanzhi Cui \\ Tumour Institute \\ Fourth Hospital \\ of Hebei Medical \\ University \\ 169 Tianshan Rd \\ Shi Jiazhuang 050011, China \\ Phone/fax: +86 311-66696305 \\ E-mail: yzchb12@sina.com
}


complicated pathology is unclear, and more work is required to identify novel molecules that are involved in the process. Therefore, investigation of the molecular mechanisms underlying NSCLC tumourigenesis may aid in the development of novel therapeutic targets and strategies for the treatment of the malignancy.

Some studies using deep transcriptome sequencing and microarrays have led to the estimation that $70-90 \%$ of the human genome is transcribed into non-protein-coding RNA [4-6]. Small non-coding RNAs such as microRNAs have been studied extensively, and their roles in gene regulation and cell function have been elucidated in numerous cancers [7-9]. Long non-coding RNAs (IncRNAs) are non-coding RNAs of more than 200 nucleotides (nt) in length and are characterised by diverse and complex sequences and mechanisms of action. Recent studies indicate that IncRNAs are involved in a variety of biological processes and diseases in humans, including cancers. Thus, IncRNAs have emerged as a new regulator in cancer research, and several studies have shown that some IncRNAs function as oncogenes, tumour suppressor genes, or both, depending on the circumstances [10-13].

Several IncRNAs have been reported to be involved in glioma. HOTAIR, a cell cycle-associated IncRNA, has been revealed to primarily serve as a prognostic factor for glioma patient survival, as well as a biomarker for identifying glioma molecular subtypes [14]. LncRNA HULC enhances epithelial-mesenchymal transition to promote tumorigenesis and metastasis of HCC via the miR-141-3p-3p/ ZEB1 signalling pathway. LncRNA BANCR expression was remarkably increased in HCC tissues; BANCR downregulation in Hep3B cells impaired cell proliferation, promoted cell apoptosis, reduced cell invasion and migration, led to downregulated venetian, and unregulated E-cadherin protein levels [15-17]. TP73-AS1, a IncRNA transcribed from chromosome1p36, has been reported to be associated with cell proliferation and tumour progress. Previous studies predicted that TP73-AS1 might be up-regulated in HCC cells through a bioinformatics database [18-20]. Although emerging evidence has suggested that TP73-AS1 associated with cancer progression and prognosis, the detailed role of TP73-AS1 in NSCLC and the underlying mechanism remains unclear.

Several studies have indicated that some specific IncRNAs can function as miRNA sponges to control miRNAs available for binding with their targets, functionally liberating mRNA transcripts targeted by certain miRNAs [21-25]. Actually, one miRNA may have multiple mRNA targets, so it may be involved in diverse pathological processes. Meanwhile, the 3 -UTR of target mRNA can be recognised by multiple miRNAs, and they can alter cellular responses via a coordinated network. Several miRNAs (miR-133b, miR-34, miR-107, miR-433 and miR-205, miR-141-3p) have been observed to be aberrantly expressed in cancer tissue and are involved in regulation of multiple biological processes such as cell proliferation, apoptosis, migration, and invasion [26-29].

In the current study, we investigate the interaction between TP73-AS1 and miR-141-3p, which can regulate NSCLC cell growth. Our findings provide a novel understanding of the TP73-AS1 and miR-141-3p axis in NSCLC cell proliferation and the molecular mechanism involved in the process of tumorigenesis.

\section{Material and methods}

\section{Clinical specimens}

Fifty-five paired NSCLC specimens and corresponding adjacent non-tumour tissues were recruited from tumour surgical resection in the Fourth Hospital of Hebei Medical University from February 2012 to December 2016. The adjacent non-tumour tissues were pathologically evaluated and in all cases they were lung tissue. None of these NSCLC patients were treated with radiotherapy, chemotherapy, or immunotherapy prior to operation. After surgery was completed, all of the tissue samples were immediately snap-frozen in liquid nitrogen and then stored at $-80^{\circ} \mathrm{C}$ until use.

The Medical Ethics Committee of the Fourth Hospital of Hebei Medical University approved this study. Written, informed consent conforming to the tenets of the Declaration of Helsinki were obtained from each participant prior to the study.

\section{Cell lines}

Human NSCLC cells line such as A459 and SPCA1 and immortalised normal human bronchial epithelial cell line (16HBE) were purchased from American Type Culture Collection (ATCC, Manassas, VA, USA). A459 and SPC-A1 were cultured in Dulbecco's modified Eagle's medium (DMEM) (Gibco Co., USA) including $10 \%$ foetal bovine serum (FBS) (Gibco Co., USA), $100 \mathrm{U} / \mathrm{ml}$ penicillin, and $100 \mathrm{ug} /$ $\mathrm{ml}$ streptomycin. $16 \mathrm{HBE}$ cell line was maintained in cell culture medium consisting of 1: 1 Medium 199 and MCDB105 medium (Sigma-Aldrich) with 10\% FBS and $10 \mathrm{ng} / \mathrm{ml}$ EGF (Sigma-Aldrich). All cells were cultured at $37^{\circ} \mathrm{C}$ in a humidified atmosphere of $5 \% \mathrm{CO} 2$ on $0.1 \%$ gelatine-coated culture flasks.

\section{Cell transfection}

Both the miRNA mimics, siRNAs, and negative controls were synthesised by Ruibo Company (Guangzhou, China). Oligonucleotide and plas- 
mid transfection were conducted by using Lipofectamine $^{\mathrm{TM}} 2000$ transfection reagent (Invitrogen, USA) followed by the protocol recommended by the manufacturer. Cells were plated in six-well plates or 96-well plates, transfected, incubated for $48 \mathrm{~h}$, and used for further assays or RNA/protein extraction.

\section{BrdU cell proliferation assay}

DNA synthesis in proliferating cells was determined by measuring 5-bromo-2-deoxyuridine (BrdU) incorporation. BrdU assays were performed at $48 \mathrm{~h}$ after transfecting A459 and SPC-A1 cells with si-NC or si-TP73-AS1. After seeding the infected cells in 96-well culture plates at a density of $2 \times 10^{3}$ cells/well, they were cultured for $24 \mathrm{~h}$ or $48 \mathrm{~h}$, and incubated with a final concentration of $10 \mu \mathrm{M}$ BrdU (BD Pharmingen, San Diego, CA, USA) for $2 \mathrm{~h}$ to $24 \mathrm{~h}$. When the incubation period ended, the medium was removed, the cells were fixed for 30 min at RT, incubated with peroxidase-coupled anti-BrdU-antibody (Sigma-Aldrich) for $60 \mathrm{~min}$ at RT, washed three times with PBS, incubated with peroxidase substrate (tetramethylbenzidine) for $30 \mathrm{~min}$, and the absorbance values were measured at $450 \mathrm{~nm}$. Background BrdU immunofluorescence was determined in cells not exposed to BrdU but stained with the BrdU antibody.

\section{RNA extraction and Quantitative RT-PCR}

Total RNA was extracted with TRIzol reagent (Life Technologies, Carlsbad, CA, USA) according to a previous report. The resulting RNA pellet was dissolved in $20 \mathrm{ml}$ of RNase-free water and then stored at $-80^{\circ} \mathrm{C}$ for subsequent examination. Then the RNA sample reverse transcriptions were synthesised for IncRNA and miRNA expression assays from total RNA of the same sample in a $30 \mu \mathrm{l}$ volume, including $2 \mu \mathrm{l}$ total RNA, $2 \mu \mathrm{l}$ reverse transcription primer (random primers for U6 snRNA and Bulge-LoopTM miRNA, RiboBio, China), $5 \mu \mathrm{l}$ RT buffer, $0.5 \mu$ l PrimeScript ${ }^{\mathrm{TM}}$ II Reverse Transcriptase $(200 \mathrm{U} / \mu \mathrm{l}), 0.5 \mu \mathrm{l}$ RNase inhibitor (40 U/

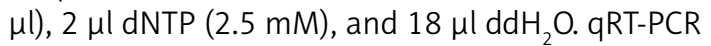
was done by using qRT-PCR using the $7500 \mathrm{HT}$ Fast Real-Time PCR System. Each reaction was carried in a total volume of $20 \mu \mathrm{l}$ including $1 \mu \mathrm{l}$ CDNA, $10 \mu \mathrm{l}$ SYBR Premix Ex TaqÔ II (TaKaRa Biotechnology Co. Ltd., China), $0.5 \mu \mathrm{l} /$ primer, and $9 \mu \mathrm{lddH} 2 \mathrm{O}$, where the program was set to $95^{\circ} \mathrm{C}$ in 5 min for pre-incubation, 40 cycles at $95^{\circ} \mathrm{C}$ in 5 seconds and at $60^{\circ} \mathrm{C}$ in $20 \mathrm{~s}$ for the annealing and amplification, as well as final addition dissociation curve. The following primers were used to detect the expression of TP73-AS1 and GAPDH (internal control): TP73AS1 F: 5'-CCGGTTTTCCAGTTCTTGCAC-3', TP73-AS1 R: 5'-GCCTCACAGGGAAACTTCATGC-3', GAPDH F: 5'-AGAAGGCTGGGGCTCATTTG-3', and GAPDH R:
5'-AGGGGCCATCCACAGTCTTC-3, miR-141-3p RT: GTCGTATCCAGTGCAGGGTCCGAGGTATTCGCACTGGATACGACTCCAAC, F: GGCGCATCTTCCAGTACAGT, U6 F: CTCGCTTCGGCAGCACA R: AACGCTTCACGAA TTTGCGT. The relative expression level of miRNAs was normalised to U6 small nucleolar RNA. All qRT-PCR runs were repeated in three replications. The results were calculated with the $2^{-\Delta \Delta C t}$ method.

\section{Dual luciferase reporter assay}

HEK293 cells were seeded into a 24-well plate. Wild-type and mutated TP73-AS1 (wt-TP73-AS1 and mut-TP73-AS1 containing an 8 bp mutation in the predicted binding sites of miR-141) luciferase reporter gene vectors were constructed based on the guidelines of the QuikChange Lighting Site-Directed Mutagenesis Kit (Stratagene, La Jolla, CA, USA). miRNA mimics and their negative control (NC) were synthesised by Guangzhou Rui Bo Biological Technology Co. Ltd., China. For the luciferase reporter detection, HEK293T cells were planted in RPMI media containing $10 \%$ foetal bovine serum at $5 \times 10^{4}$ cells per well in 24-well plates. After $24 \mathrm{~h}$, these cells were co-transfected with 50 ng psiCHECK23'UTR wild-type or mutant reporter plasmids. In the meantime, these wells of cell culture were added by $100 \mathrm{nM}$ of miRNAs mimic or miR-NC by using Lipofectamine 2000 transfection reagent (Invitrogen, Carlsbad, CA, USA). The activities of firefly and Renilla luciferases were assessed after $48 \mathrm{~h}$ by using Dual-Glo ${ }^{\circledast}$ Luciferase Assay System (Promega, Cat. E2920, USA), based on the manufacturer's protocols. Each treatment was performed in triplicate in three independent experiments.

\section{Statistical analysis}

The results are shown as the mean \pm SEM. The Welch's corrected unpaired $T$ test and the Kruskal-Wallis test were used to compare continuous variables. The $\chi^{2}$ test and Fisher's exact test were used to compare categorical variables. The relative expression of miR-141-3p was calculated using the $2^{-\Delta \Delta C t}$ method, whereby the average cycle threshold $(C t)$ values of miR-141-3p were subtracted from the $C t$ values of U6. $P$-values less than 0.05 were considered statistically significant. All statistical analyses were performed using SPSS (Statistical Package for the Social Sciences) software (version 18.0; SPSS Inc.).

\section{Results}

The level of TP73-AS1 was increased in NSCLC tissues and cell lines

Initially the expression levels of TP73-AS1 in 55 paired samples (NSCLC specimens and corresponding adjacent non-tumour tissues) were ex- 
amined using real-time PCR. Results indicated that TP73-AS1 expression was significantly unregulated in tumour tissues compared that of paired normal tissues (Figure $1 \mathrm{~A}$ ). Then TP73-AS1 expressions in two human NSCLC cell lines A459 and SPC-A1 and immortalised normal human bronchial epithelial cell line (16HBE) as control were determined using real-time PCR. Results suggested that the levels of TP73-AS1 expression were also at higher levels in A459 and SPC-A1 cell lines, compared to the control group (Figure $1 \mathrm{~B}$ ). These data provide potential evidence that TP73-AS1 was more expressed in NSCLC tissues and cell lines and may be related to poorer prognosis; however, the detailed mechanism underlying the action of TP73-AS1 on NSCLC remains unclear.

\section{TP73-AS1 knockdown suppressed NSCLC cell proliferation}

Higher IncRNA TP73-AS1 levels in both NSCLC tissues and cell lines as compared to non-cancer controls suggested that IncRNA TP73-AS1 might play a key role in NSCLC tumourigenesis. To investigate the detailed functions of TP73-AS1 in NSCLC, we generated a series of functional assays. We designed IncRNATP73-AS1 siRNA1, which we transfected into A459 and SPC-A1 cells to achieve TP73-AS1 knockdown, as verified using real-time PCR assays (Figure 2 A). After TP73-AS1 was knocked down, the cell proliferation was moni- tored with use of BrdU. As shown in Figure $2 \mathrm{~B}$, the proliferation of A459 and SPC-A1 cells was significantly down-regulated by TP73-AS1 knockdown compared to the si-NC (negative control) group.

\section{LncRNA TP73-AS1 interacted with miR-141-3p}

Recently, it has been reported that IncRNAs can act as miRNA sponges, reducing their regulatory effect on mRNAs. This function introduces an extra layer of layer of complexity in the miRNA-target interaction network. Dysfunction of non-coding RNAs, mainly IncRNAs and miRNAs, can affect processes, including cancer cell proliferation, apoptosis, invasion, and the metastatic process in cancer's complexity in the miRNA-target interaction network. Among well-established miRNAs in cancers, miR-141 plays an essential role in the regulation of cancer cell proliferation. Furthermore, we employed luciferase assays to confirm the miR-141 regulation of TP73-AS1. A wild-type and mutated TP73-AS1 (wt-TP73-AS1and mut-TP73AS1 containing an 8 bp mutation in the predicted binding sites of miR-142) luciferase reporter gene vector was constructed (Figure $3 \mathrm{~A}$ ). These indicated vectors were co-transfected into HEK293 cells with miR-142 mimics or miR-142 NC; the luciferase activity was then monitored. Results showed that the luciferase activity of wt-TP73-AS1 vector could be significantly suppressed by miR-141
A

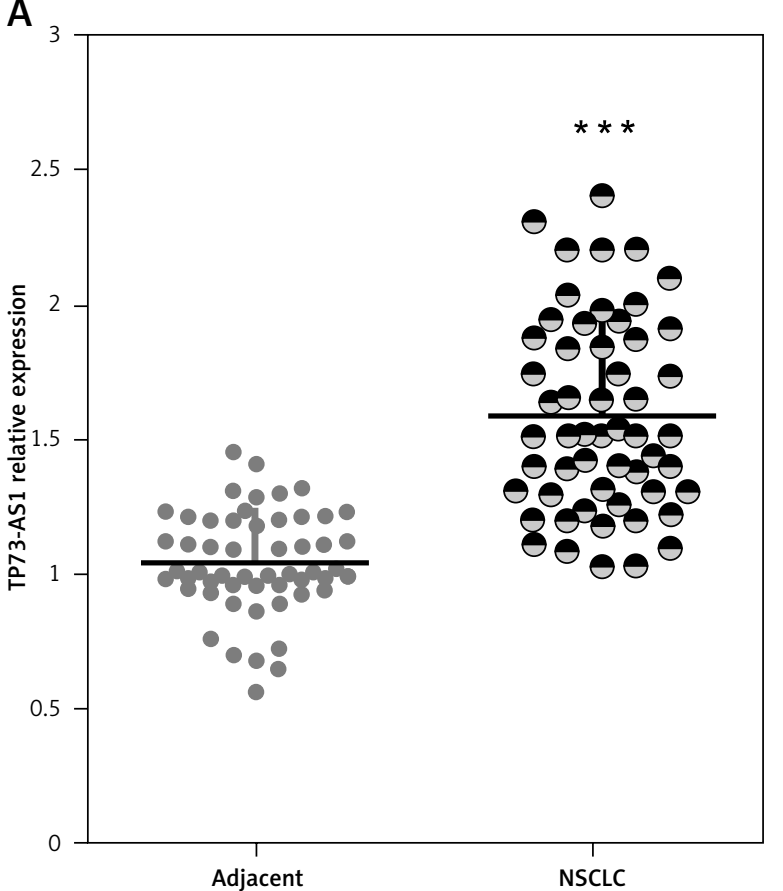

B

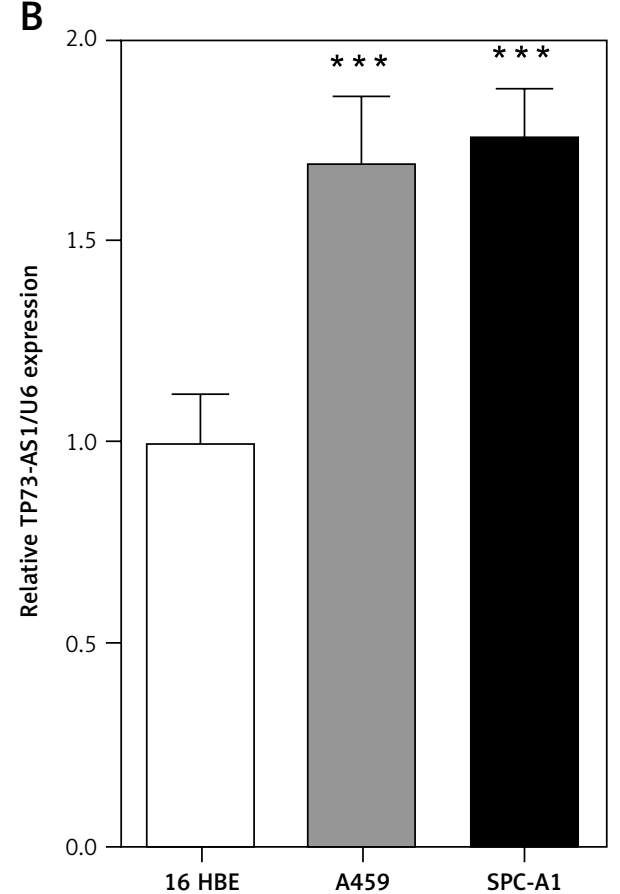

Figure 1. High TP73-AS1 expression in NSCLC tissues and cell lines. A - A total of 55 paired NSCLC tissues and tumour tissues were monitored for TP73-AS1 expression using real-time PCR assays. B - TP73-AS1 expression in NSCLC cell lines, A459, SPC-A1 and a normal cell line, using real-time PCR assays. The data are presented as mean \pm SD of three independent experiments ${ }^{*} p<0.05,{ }^{* *} p<0.01,{ }^{* * *} p<0.001$. 
A

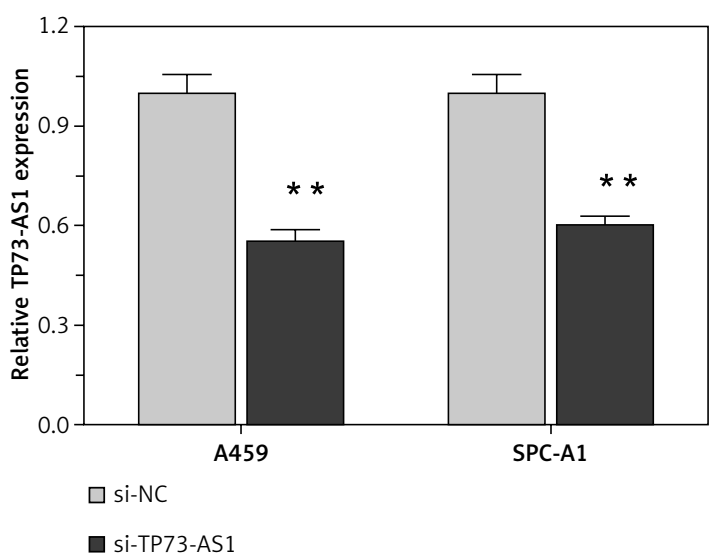

B

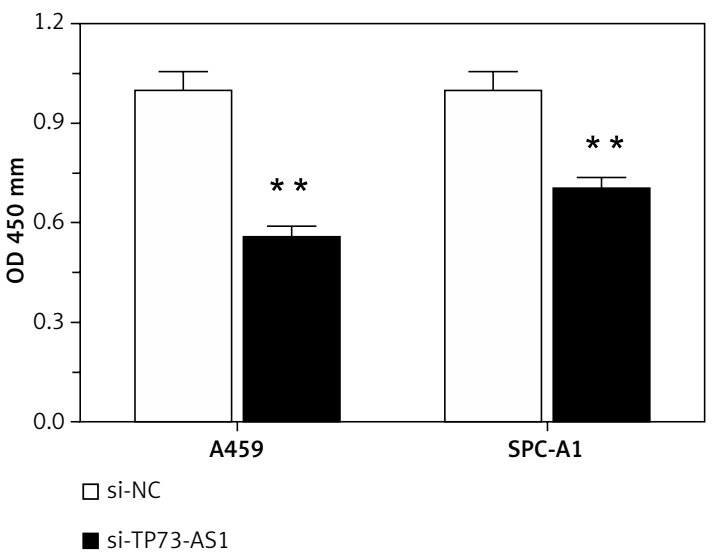

Figure 2. TP73-AS1 knockdown inhibited NSCLC cell proliferation. A - si-TP73-AS1 was transfected into A459 and SPC-A1 cells to achieve TP73-AS1 knockdown, as verified using real-time PCR. B - After si-TP73-AS1 transfection, the cell viability of A459 and SPC-A1 was determined using BrdU assays. The data are presented as mean \pm SD of three independent experiments ${ }^{*} p<0.05,{ }^{* *} p<0.01$.

A

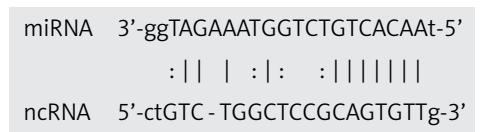

B

C
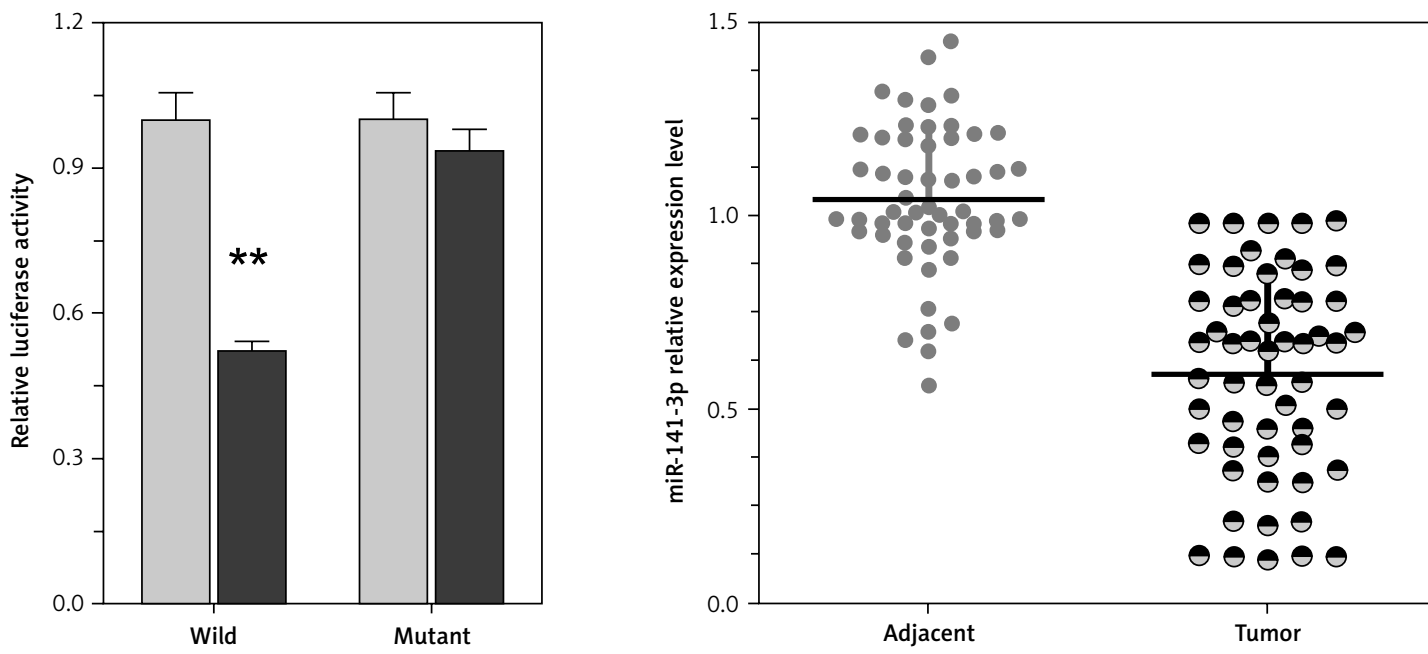

$\square$ miR-NC

$\square$ miR-141-3p mimic

Figure 3. LncRNA TP73-AS1 interacted with miR-141-3p. A - A wild-type and mutated TP73-AS1 (wt-TP73-AS1 and mut-TP73-AS1 containing an 8 bp mutation in the predicted binding sites of miR-141) luciferase reporter gene vector was constructed. B - After overnight culture, cells were co-transfected with the indicated vectors and miR-141 mimics. Luciferase assays were performed $48 \mathrm{~h}$ after transfection using the Dual Luciferase Reporter Assay System to determine the luciferase activity. C - Expression levels of miR-141-3p in a total of 55 paired tumours was monitored using real-time PCR assays. The data are presented as mean \pm SD of three independent experiments ${ }^{*} p<0.05,{ }^{* *} p<0.01$.

mimics; after mutation in the predicted binding sites of miR-141 in TP73-AS1, the effect of miR141 mimics or miR-141 NC on luciferase activity was abolished (Figure $3 \mathrm{~B}$ ), indicating that TP73AS1 could directly bind to miR-141. We revealed that TP73-AS1 might act as a competing endoge- nous RNA (ceRNA) to promote NSCLC cell proliferation by sponging miR-141. Finally, we evaluated the expression levels of TP73-AS1 and miR-142 in tumour tissues. As exhibited by real-time PCR assays, miR-141 expression was significantly down-regulated in NSCLC tissues (Figure $3 \mathrm{C}$ ). 


\section{Discussion}

Studies have demonstrated that among ncRNAs, $\sim 18 \%$ of IncRNAs are associated with human tumours, compared with only $9 \%$ of human protein-coding genes, suggesting that IncRNAs could act as major contributors to carcinogenesis and cancer progression [30, 31]. Many ncRNAs show abnormal expression patterns in cancerous tissues. These include miRNAs, long mRNA-like nCRNAs, GAS5, SNORD50, telomerase RNA, and $Y$ RNAs [32-35]. The miRNAs are involved in the large-scale regulation of many protein coding genes; the $Y$ RNAs are important for the initiation of DNA replication, telomerase RNA that serves as a primer for telomerase, an RNP that extends telomeric regions at chromosome ends (see Telomeres and disease for more information). The direct function of the long mRNA-like ncRNAs is less clear.

LncRNA TP73-AS1 is located on human chromosomal band 1p36.32. TP73-AS1 is the antisense of the coding gene TP73, which encodes a product that shares structural and functional characteristics with TP53. Global genomic analysis has shown that up to $70 \%$ of protein-coding transcripts have antisense partners, and the perturbation of the antisense RNA alters the expression of the sense gene. TP73-AS1 covers substantial portions of TP73, suggesting that TP73-AS1 may function by posttranscriptional regulation of TP73 gene expression. LncRNA TP73-AS1 has been reported to be upregulated in oesophageal squamous cell carcinoma [18-20]. LncRNA TP73-AS1 knockdown inhibited BDH2 expression in EC9706 and KYSE30 cells, whereas $\mathrm{BDH} 2$ knockdown repressed oesophageal cancer cell proliferation and induced apoptosis via the caspase-3-dependent apoptotic pathway [19]. To the best of our knowledge, information on InCRNA TP73-AS1 in NSCLC is still limited.

In the current study, we initially monitored TP73-AS1 expression in a large panel of 55 paired NSCLC tissues and adjacent normal tissues. A significantly higher expression level of TP73-AS1 in NSCLC tissues was observed compared to the adjacent normal tissues. Consistently, the expression level of TP73-AS1 was obviously higher in NSCLC cell lines compared to that of the normal cell lines. Then, we designed IncRNA TP73-AS1 siRNA to block TP73-AS1 expression, and thus attenuate proliferation of NSCLC. We found that InCRNA TP73-AS1 siRNA inhibits NSCLC cell proliferation. These results are in agreement with the findings of Yang, et al., who reported that InRNA TP73-AS1 is significantly downregulated in NSCLC as compared to normal lung tissues ( $p<0.001$ ), but it is strongly unregulated in large-cell carcinoma specimens compared to adenocarcinoma, smallcell lung cancer, and squamous cell carcinoma tissues [36]. These findings indicate that InRNA
TP73-AS1 may play an important role in the development and progression of various tumours.

In recent years, it has been proven that in a variety of cell processes, IncRNAs played a vital role by acting as ceRNAs to mediate the miRNAs. Hamada et al. demonstrated that a deregulated triplet, composed of mRNA (RFX1), IncRNA (TP73AS1), and miRNA (miR-197), contributed to the development and progression of glioblastoma multiforme [37]. These studies suggest that this IncRNA-microRNA interaction plays an important role in cancer progression through regulation of cancer growth and metastasis. In the present study, we investigated the interaction between TP73-AS1 and miR-141-3p for the first time: TP73AS1 and miR-141-3p could dually regulate each other in a negatiThe miR-141-3p is believed to play an essential role in tumour suppression by inhibiting epithelial-mesenchymal transition (EMT), the initiating step of metastasis (Korpal). EMT occurs as part of embryonic development and shares many similarities with cancer progression [38-40]. During EMT, cells lose adhesion and increase in motility. This is characterised by repression of

E-cadherin expression, which also occurs during the initial stages of metastasis [41-43]. By contrast, miR-141-3p has been shown to promote the last step of metastasis in which migrating cancer cells undergo MET during their colonisation at distant tissues. In a series of mouse mammary isogenic cancer cell lines, the miR-141-3p family is highly expressed only in the cells that are able to form metastases (4T1 cells) but not in other cells that are unable to colonise (4TO7 cells). Overexpression of miR-141-3pc in non-metastatic 4TO7 cells readily enables MET and colonisation of the liver and lung. miR-14-3p targets the E-cadherin transcriptional repressors ZEB1 and ZEB2. Knockdown of miR-141-3p and miR-200b has been shown to reduce $\mathrm{E}$-cadherin expression thus increasing cell motility and inducing EMT [44-47].

In conclusion, the TP73-AS1/miR-142 axis may play a key role in NSCLC cell proliferation and may provide a therapeutic application in NSCLC patients.

\section{Acknowledgments}

Xinfa Liu and Mingwing Wang equally contributed and should be considered as co-first authors.

\section{Conflict of interest}

The authors declare no conflict of interest.

\footnotetext{
References

1. Siegel RL, Miller KD, Jemal A. Cancer statistics, 2016. CA Cancer J Clin 2016; 66: 7-30.

2. Park C, Lee IJ, Jang SH, Lee JW. Factors affecting tumor recurrence after curative surgery for NSCLC: impacts
} 
of lymphovascular invasion on early tumor recurrence. J Thorac Dis 2014; 6: 1420-8.

3. Sherwood J, Dearden S, Ratcliffe M, Walker J. Mutation status concordance between primary lesions and metastatic sites of advanced non-small-cell lung cancer and the impact of mutation testing methodologies: a literature review. J Exp Clin Cancer Res 2015; 34: 92.

4. Chen J, Hu L, Wang J, et al. Low expression LncRNA TUBA4B is a poor predictor of prognosis and regulates cell proliferation in non-small cell lung cancer. Pathol Oncol Res 2017; 23: 265-70.

5. Zequn N, Xuemei Z, Wei L, et al. The role and potential mechanisms of LnCRNA-TATDN1 on metastasis and invasion of non-small cell lung cancer. Oncotarget 2016; 7: 18219-28.

6. Nie W, Ge HJ, Yang XQ, et al. LnCRNA-UCA1 exerts oncogenic functions in non-small cell lung cancer by targeting miR-193a-3p. Cancer Lett 2016; 371: 99-106.

7. Hu X, Bao J, Wang Z, et al. The plasma IncRNA acting as fingerprint in non-small-cell lung cancer. Tumour Biol 2016; 37: 3497-504.

8. Li P, Li J, Yang R, et al. Study on expression of IncRNA RGMB-AS1 and repulsive guidance molecule $b$ in nonsmall cell lung cancer. Diagn Pathol 2015; 10: 63.

9. Zhao JJ, Chen PJ, Duan RQ, Li KJ, Wang YZ, Li Y. miR-630 functions as a tumor oncogene in renal cell carcinoma. Arch Med Sci 2016; 12: 473-8.

10. Xie X, Liu HT, Mei J, et al. LncRNA HMlincRNA717 is down-regulated in non-small cell lung cancer and associated with poor prognosis. Int J Clin Exp Pathol 2014; 7: 8881-6.

11. Yang YR, Zang SZ, Zhong CL, Li YX, Zhao SS, Feng XJ. Increased expression of the IncRNA PVT1 promotes tumorigenesis in non-small cell lung cancer. Int J Clin Exp Pathol 2014; 7: 6929-35.

12. Schmidt LH, Gorlich D, Spieker T, et al. Prognostic impact of Bcl-2 depends on tumor histology and expression of MALAT-1 IncRNA in non-small-cell lung cancer. J Thorac Oncol 2014; 9: 1294-304.

13. Yang Y, Li H, Hou S, Hu B, Liu J, Wang J. The noncoding RNA expression profile and the effect of IncRNA AK126698 on cisplatin resistance in non-small-cell lung cancer cell. PLoS One 2013; 8: e65309.

14. Huang K, Sun J, Yang C, et al. HOTAIR upregulates an 18-gene cell cycle-related mRNA network in glioma. Int J Oncol 2017. doi: 10.3892/ijo.2017.3901.

15. Xiong $\mathrm{H}, \mathrm{Ni} \mathrm{Z}$, He J, et al. LncRNA HULC triggers autophagy via stabilizing Sirt1 and attenuates the chemosensitivity of HCC cells. Oncogene 2017; 36: 3528-40.

16. Li SP, Xu HX, Yu Y, et al. LncRNA HULC enhances epithelial-mesenchymal transition to promote tumorigenesis and metastasis of hepatocellular carcinoma via the miR-200a-3p/ZEB1 signaling pathway. Oncotarget 2016; 7: 42431-46.

17. Zhao J, Fan Y, Wang K, et al. LncRNA HULC affects the differentiation of Treg in HBV-related liver cirrhosis. Int Immunopharmacol 2015; 28: 901-5.

18. Li S, Huang Y, Huang Y, et al. The long non-coding RNA TP73-AS1 modulates HCC cell proliferation through miR-200a-dependent HMGB1/RAGE regulation. J Exp Clin Cancer Res 2017; 36: 51.

19. Zhang R, Jin H, Lou F. The long non-coding RNA TP73AS1 interacted with miR-142 to modulate brain glioma growth through HMGB1/RAGE pathway. J Cell Biochem 2018; 119: 3007-16.

20. Zang W, Wang T, Wang $Y$, et al. Knockdown of long non-coding RNA TP73-AS1 inhibits cell proliferation and induces apoptosis in esophageal squamous cell carcinoma. Oncotarget 2016; 7: 19960-74.

21. Ahmad A, Maitah MY, Ginnebaugh KR, et al. Inhibition of Hedgehog signaling sensitizes NSCLC cells to standard therapies through modulation of EMT-regulating miRNAs. J Hematol Oncol 2013; 6: 77.

22. Wang DT, Ma ZL, Li YL, et al. miR-150, p53 protein and relevant miRNAs consist of a regulatory network in NSCLC tumorigenesis. Oncol Rep 2013; 30: 492-8.

23. Ma K, Xu A, Cui S, Sun MR, Xue YC, Wang JH. Impaired GABA synthesis, uptake and release are associated with depression-like behaviors induced by chronic mild stress. Transl Psychiatry 2016; 6: e910.

24. Ma K, Guo L, Xu A, Cui S, Wang JH. Molecular mechanism for stress-induced depression assessed by sequencing miRNA and mRNA in medial prefrontal cortex. PLoS One 2016; 11: e0159093.

25. Ma K, Zhang H, Baloch Z. Pathogenetic and therapeutic applications of tumor necrosis factor-alpha (TNF-alpha) in major depressive disorder: a systematic review. Int J Mol Sci 2016; 17. pii: E733.

26. Asci R, Vallefuoco F, Andolfo I, Bruno M, De Falco L, Iolascon A. Trasferrin receptor 2 gene regulation by microRNA 221 in SH-SY5Y cells treated with MPP(+) as Parkinson's disease cellular model. Neurosci Res 2013; 77: 121-7.

27. Alvarez-Erviti L, Seow Y, Schapira AH, Rodriguez-Oroz MC, Obeso JA, Cooper JM. Influence of microRNA deregulation on chaperone-mediated autophagy and alpha-synuclein pathology in Parkinson's disease. Cell Death Dis 2013; 4: e545.

28. Zhang X, Guo J, Ai S, et al. Mutation analysis of micro RNA-7 gene in Chinese patients with Parkinson's disease. Zhong Nan Da Xue Xue Bao Yi Xue Ban 2012; 37: 1189-92.

29. Cho HJ, Liu G, Jin SM, et al. MicroRNA-205 regulates the expression of Parkinson's disease-related leucine-rich repeat kinase 2 protein. Hum Mol Genet 2013; 22: 608-20.

30. Chang L, Li C, Lan T, et al. Decreased expression of long non-coding RNA GAS5 indicates a poor prognosis and promotes cell proliferation and invasion in hepatocellular carcinoma by regulating vimentin. Mol Med Rep 2016; 13: 1541-50.

31. Hu L, Ye H, Huang G, et al. Long noncoding RNA GAS5 suppresses the migration and invasion of hepatocellular carcinoma cells via miR-21. Tumour Biol 2016; 37: 2691-702.

32. Soeno Y, Fujita K, Kudo T, et al. Generation of a mouse model with down-regulated U50 snoRNA (SNORD50) expression and its organ-specific phenotypic modulation. PLoS One 2013; 8: e72105.

33. Liang W, Lv T, Shi X, et al. Circulating long noncoding RNA GAS5 is a novel biomarker for the diagnosis of nonsmall cell lung cancer. Medicine (Baltimore) 2016; 95: e4608.

34. Mayama T, Marr AK, Kino T. Differential expression of glucocorticoid receptor noncoding RNA repressor Gas5 in autoimmune and inflammatory diseases. Horm Metab Res 2016; 48: 550-7.

35. Qian X, Xu C, Zhao P, Qi Z. Long non-coding RNA GAS5 inhibited hepatitis $C$ virus replication by binding viral NS3 protein. Virology 2016; 492: 155-65.

36. Yu H, Xu Q, Liu F, Ye X, Wang J, Meng X. Identification and validation of long noncoding RNA biomarkers in human non-small-cell lung carcinomas. J Thorac Oncol 2015; 10: 645-54.

37. Hamada S, Satoh K, Miura S, et al. miR-197 induces epithelial-mesenchymal transition in pancreatic cancer 
cells by targeting p120 catenin. J Cell Physiol 2013; 228: 1255-63.

38. Liu CZ, Ye ZH, Ma J, et al. A qRT-PCR and gene functional enrichment study focused on downregulation of miR141-3p in hepatocellular carcinoma and its clinicopathological significance. Technol Cancer Res Treat 2017; 16: 835-49.

39. Lei $K$, Liang $X$, Gao $Y$, et al. Lnc-ATB contributes to gastric cancer growth through a MiR-141-3p/TGFbeta2 feedback loop. Biochem Biophys Res Commun 2017; 484 514-21.

40. Capri M, Olivieri F, Lanzarini $C$, et al. Identification of miR-31-5p, miR-141-3p, miR-200c-3p, and GLT1 as human liver aging markers sensitive to donor-recipient age-mismatch in transplants. Aging Cell 2017; 16: 262-72.

41. Li JZ, Li J, Wang HQ, Li X, Wen B, Wang YJ. MiR-141-3p promotes prostate cancer cell proliferation through in hibiting kruppel-like factor-9 expression. Biochem Biophys Res Commun 2017; 482: 1381-6.

42. Verrando P, Capovilla M, Rahmani R. Trans-nonachlor decreases miR-141-3p levels in human melanocytes in vitro promoting melanoma cell characteristics and shows a multigenerational impact on miR-8 levels in Drosophila. Toxicology 2016; 368-369: 129-41.

43. Liep J, Kilic E, Meyer HA, Busch J, Jung K, Rabien A. Cooperative effect of miR-141-3p and miR-145-5p in the regulation of targets in clear cell renal cell carcinoma. PloS One 2016; 11: e0157801.

44. Li D, Li J. Association of miR-34a-3p/5p, miR-141-3p/5p, and miR-24 in decidual natural killer cells with unexplained recurrent spontaneous abortion. Med Sci Monit 2016; 22: 922-9.

45. Ji J, Qin Y, Ren J, et al. Mitochondria-related miR-141-3p contributes to mitochondrial dysfunction in HFD-induced obesity by inhibiting PTEN. Sci Rep 2015; 5: 16262.

46. Wang C, Ouyang Y, Lu M, Wei J, Zhang H. miR-141-3p regulates the expression of androgen receptor by targeting its 3'UTR in prostate cancer LNCaP cells. Xi Bao Yu Fen Zi Mian Yi Xue Za Zhi 2015; 31: 736-9.

47. Qiu W, Kassem M. miR-141-3p inhibits human stromal (mesenchymal) stem cell proliferation and differentiation. Biochim Biophys Acta 2014; 1843: 2114-21. 